\title{
World ORS Day 2019: Save The Children Save Generation Next
}

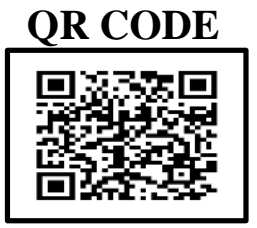

In many developing nations, one of the leading cause of infant mortality are acute diarrheal diseases. According to the $\mathrm{WHO}$, diarrheal diseases stand second in line of most common causes of under 5 year mortality. Diarrheal diseases lead to fluid loss from the body further reaching a state of severe dehydration. This complication may be fatal or potentially life-threatening. Oral Rehydrating Solution (ORS) is an electrolyte-based solution which is used for keeping the body in a hydrated state and helps in rehydrating the affected subjects by a mechanism that basically involves compensation of the loss of salts, minerals and body fluids. The WHO and UNICEF recommend that an integrated approach should be adopted to prevent and treat diarrheal diseases and their complications. WASH (water, sanitation and hygiene) education, timely vaccination, proper nutrition, appropriate breastfeeding and treatment with ORS and Zinc are the tools that help to achieve this goal.

In recent decades, there has been a decline in global deaths from childhood diarrhoea, but still the toll hits up to a total of half a million annually. A prime contribution to this drop was given by the use of ORS for both prevention and treatment of dehydration. It was back in 1978, when WHO launched the global diarrheal control programme that aimed to decrease mortality due to diarrheal causes with ORS at its center and it was addressed as 'potentially the most important medical advance' of the zoth century. After a swift intensification by the WHO and other international agencies, ORS is currently being used for treatment and prevention in about one third of diarrheal diseases. ${ }^{1}$

ORS day is observed and celebrated on July $29^{\text {th }}$ every year aiming to protect young children from acute diarrheal conditions and dehydration. In the year 2002, Norbett Hirschhorn, Nathaniel Pierce, Dilip Mahalanabis, and David Nalin received the first Pollin prize in pediatric research for their work on ORS as well as creation of awareness around it. ${ }^{2}$
Identification of the dehydrating symptoms, ensuring adequate water consumption, constant urination to get rid of toxins, fats and bodily wastes and drinking plenty of potable water for hydration of body are the objectives to be achieved in order to comply with the goals of ORS day. ${ }^{3}$

\section{Using packaged ORS powder:}

- Pre-packed formula called Oral Rehydration Salts is readily available at medical stores, clinics and healthcare units. It is being sold with countless trade names and a variety of flavors. It is mandatory to check the packet for directions of use stated by the concerned manufacturer. If not detailed, one should follow steps mentioned below.

- Add the contents of the packet to a glass or container. Pour a little amount of clean water. ORS should not be added to juice, milk, soft drinks or any other kind of drink.

- Stir the solution thoroughly and serve it to the child.

- There is no need to add sugar or salt to the prepacked mixture as it already contains the required concentration of the same.

\section{Preparing ORS at home:}

It requires one litre of clean water fit for drinking, six teaspoons of sugar and half a teaspoon of salt. Mix all the ingredients and stir the mixture thoroughly until the dissolution of sugar. Addition of too much sugar should be avoided it lead to worsening of the diarrheal problems. The prepared solution is to be kept covered and should not be stored for more than 24 hours. It is advisable to prepare a fresh mixture every day. Also make sure that the water used to prepare ORS should be clean as contaminated water does not benefit the process of fluid replacement. Moreover, bacterial contamination can further cause a progression in the severity of diarrheal diseases. ${ }^{3}$ 
Another critical new advancement in the treatment of diarrheal episodes is the administration of Zinc as oral Zinc. WHO has added recently co-packaged oral rehydration solution and zinc to its Model List of Essential Medicines. ${ }^{4}$ Recent clinical trials have proved that the element Zinc boosts up and fortifies the human immune system and helps in replenishment of depleted nutrients children recover faster from the disease by and fortifying the immune system. It has been proven to prevent future episodes of diarrhea too. ${ }^{5}$

\section{Contribute to ORS day:}

Individuals can contribute to the health of the children as a whole by gaining knowledge about causes, symptoms, prevention and treatment of diarrheal diseases and dehydration and spreading this to the community and fellow workers via social media and other modes of communication and letting everybody know how important is the health of your child, how serious are the consequences of dehydration and how easy is to prevent and treat diarrheal episodes and dehydration by the means of ORS. Health education regarding the same is the need of the hour starting from the primordial level.

Healthcare organizations and related bodies need to frame policies and promote funding for treatment and management of dehydration and related complications.
ORS day can be considered to be the best time to help the needful children and educate the public detailing this issue and the benefits of using Oral Rehydration Solution complemented with Zinc to prevent and treat the escalating public health problem of diarrhea and dehydration.

\section{REFERENCES}

1. Black RE. Progress in the use of ORS and zinc for the treatment of childhood diarrhea. J Glob Health. 2019;9(1):010101.

2. https://www.nlm.nih.gov/exhibition/againsttheodd s/exhibit/community health/simple solution.html. [last assessed on 5th July,2019]

3. www.apollopharmacy.in/blog/world-ors-day. [last assessed on $5^{\text {th }}$ July,2019]

4. https://www.who.int/selection medicines/committ ees/expert/22/s17.5 ORS-zinc.pdf. [last assessed on $5^{\text {th }}$ July,2019]

5. Bajait C, Thawani V. Role of zinc in pediatric diarrhea. Indian J Pharmacol. 2011;43:232-5.
Cite this article as:

Jain S. World ORS Day 2019: Save The Children Save Generation Next. Int Healthc Res J. 2019;3(4):133-134. https://doi.org/10.26440/IHRJ/0304.07253 